\title{
Estimating the surgical backlog from the COVID-19 lockdown in South Africa: A retrospective analysis of six government hospitals
}

\author{
K M Chu, ${ }^{1}$ MD, MPH, FACS, FASRCS; J Marco, ${ }^{1}$ BA, MA; H Bougard, ${ }^{2}$ MB ChB, FCS(SA), FACS; C P Strauss, ${ }^{3}$ MB ChB, FCS(SA); \\ L Bertels, ${ }^{4} \mathrm{BSc}, \mathrm{MB}$ ChB, FCS(SA); A E Victor, ${ }^{5} \mathrm{MB} \mathrm{ChB}, \mathrm{FCS}(\mathrm{SA})$, MMed (General Surgery); L van der Walt, ${ }^{5} \mathrm{MB}$ ChB; \\ A Goliath, ${ }^{6} \mathrm{MB} \mathrm{ChB}, \mathrm{MMed}$ (General Surgery); R Duvenage, ${ }^{7,8} \mathrm{MB} \mathrm{ChB}, \mathrm{MMed}$ (General Surgery) \\ ${ }^{1}$ Centre for Global Surgery, Department of Global Health, Faculty of Medicine and Health Sciences, Stellenbosch University. Cape Town, \\ South Africa \\ ${ }^{2}$ Department of Surgery, New Somerset Hospital, Cape Town, South Africa \\ ${ }^{3}$ Department of Surgery, Eerste Rivier Hospital, Cape Town, South Africa \\ ${ }^{4}$ Department of Surgery, Mitchell's Plain Hospital, Cape Town, South Africa \\ ${ }^{5}$ Department of Surgery, Karl Bremer Hospital, Cape Town, South Africa \\ ${ }^{6}$ Department of Surgery, Khayelitsha District Hospital, Cape Town, South Africa \\ ${ }^{7}$ Department of Surgery, Worcester Regional Hospital, Western Cape, South Africa \\ ${ }^{8}$ Ukwanda Centre for Rural Health, Faculty of Medicine and Health Sciences, Stellenbosch University, Cape Town, South Africa
}

Corresponding author: KM Chu (kchu@sun.ac.za)

\begin{abstract}
Background. The COVID-19 pandemic reached South Africa (SA) in March 2020. A national lockdown began on 27 March 2020, and health facilities reduced non-essential activity, including many surgical services.

Objectives. Primary objective: to estimate the COVID-19 surgical backlog in Western Cape Province, SA, by comparing 2019 and 2020 general surgery operative volume and proportion at six district and regional hospitals. Secondary objective: to compare the operative volume of appendicectomy, laparoscopic cholecystectomy, cancer and trauma between the 2 years.

Methods. This was a retrospective study of general surgery operations from six SA government hospitals in the Western Cape. Data were obtained from electronic operative databases or operative theatre logbooks from 1 April to 31 July 2019 and 1 April to 31 July 2020.

Results. Total general surgery operations decreased by $44 \%$ between 2019 ( $n=3247)$ and $2020(n=1810)(p<0.001)$. Elective operations decreased by $74 \%$ ( $n=1379$ v. $n=362 ; p<0.001$ ), and one common elective procedure, laparoscopic cholecystectomy, decreased by $68 \%$ $(p<0.001)$. Emergency operations decreased by $22 \%(n=1868$ v. $n=1448 ; p<0.001)$ and trauma operations by $42 \%(n=325$ v. $n=190$; $p<0.001)$. However, non-trauma emergency operations such as appendicectomy and cancer did not decrease. The surgical backlog for elective operations after 4 months from these six hospitals is 1017 cases, which will take between 4 and 14 months to address if each hospital can do one additional operation per weekday.

Conclusions. The COVID-19 pandemic has created large backlogs of elective operations that will need to be addressed urgently. Clear and structured guidelines need to be developed in order to streamline the reintroduction of full surgical healthcare services as SA slowly recovers from this unprecedented pandemic.
\end{abstract}

S Afr Med J 2021;111(7):685-688. https://doi.org/10.7196/SAMJ.2021.v111i7.15686

In March 2020, the World Health Organization declared a pandemic due to the rapid spread of COVID-19, a disease resulting from a novel coronavirus, SARS-CoV-2. South Africa (SA) has had more than 1 million cases, more than half of the COVID-19 infection on the African continent. ${ }^{[1]}$ The first COVID-19 case in SA was on 5 March 2020. In preparation for the anticipated upsurge in cases, a national lockdown began on 27 March, which closed all borders, schools and large gatherings. All persons were required to stay at home if not involved in essential activities. Because COVID-19 is an acute respiratory syndrome, some patients with severe disease have required ventilator support and intensive care unit support. In order to prepare hospitals for the COVID-19 surge, health facilities reduced non-essential activity, such as elective surgical care. ${ }^{[2]}$ A 2020 national survey of SA surgeons reported that $>90 \%$ of hospitals cancelled or reduced elective procedures in April. ${ }^{[3]}$ Modelling studies have estimated a national backlog of $\sim 150000$ surgical procedures, but studies reporting primary data on operative volume are lacking. ${ }^{[3,4]}$

\section{Objectives}

The primary objective of this study was to estimate the surgical backlog due to the COVID-19 pandemic in Western Cape Province, SA, by comparing 2019 and 2020 elective general surgery operative volume at six district and regional hospitals. The secondary objective was to compare the operative volume of appendicectomy, laparoscopic cholecystectomy, cancer and trauma between the 2 years.

\section{Methods}

\section{Design and setting}

This was a retrospective study of general surgery operations from six SA government hospitals in the Western Cape. Data were obtained 
from electronic databases or operation theatre logbooks from 1 April to 31 July 2019 and 1 April to 31 July 2020. The study hospitals included two regional and four district hospitals (Table 1).

\section{Participants}

Patients of all ages (adults and children) who underwent a general surgery operation at one of the study hospitals were included. An operation was defined as a surgical procedure requiring an incision under anaesthesia occurring in an operating theatre.

\section{Data analysis}

Data were de-identified, then extracted from hospital databases or operation logbooks and imported into Stata 13 (StataCorp, USA) for analysis. All operations performed by the general surgery service from each hospital were included. These procedures were classified as emergency or elective. In addition, data on one common elective procedure, laparoscopic cholecystectomy, were collected as a proxy to measure elective operative volume change. While early laparoscopic cholecystectomy is advocated for acute cholecystitis, this rarely occurs in SA government hospitals owing to resource constraints, and the procedure is usually done electively. ${ }^{[5,6]}$ Procedural data for four emergency conditions (trauma, appendicitis, breast cancer and colorectal cancer) were also collected.

The surgical backlog was calculated by the difference between 2019 and 2020 elective operations. Assuming that each hospital could accommodate an additional operation during weekdays to address the backlog, the time in months to address the backlog was equal to the backlog for each hospital divided by 22 (weekdays).

Descriptive variables included patient age and gender, and year of operation. Outcomes included the number for each procedure type and the surgical backlog for elective operations. Poisson regression modelling was used to compare operative volume between years, and $p$-values $<0.05$ were considered statistically significant.

\section{Ethical considerations}

Ethics approval for the study was obtained from the Stellenbosch University Human Research Ethics Committee (ref. no. N20/08/046_COVID-19) and the Western Cape Department of Health (ref. no. WC 202008_059). Since this was a secondary analysis of de-identified data, informed consent was waived.

\section{Results}

Study population demographics between 2019 and 2020 were similar. The mean age in 2019 was 42.6 years, compared with 42.3 years in $2020(p=0.502)$. There were $46.2 \%$ females in 2019, compared with $44.8 \%$ in $2020(p=0.359)$. Total operations decreased by $42 \%$ (36 - 58\% per hospital, data not shown) from 2019 ( $n=3$ 247) to $2020(n=1810)(p<0.001)$ (Fig. 1$)$.

\section{Elective operations}

Elective operations decreased by $73 \%$ from 2019 ( $n=1379)$ to $2020(n=368)(p<0.001)$. Every study hospital had a significant decrease in elective operations (Table 2). The absolute number of laparoscopic cholecystectomies decreased by $68 \%$ between $2019(n=242)$ and $2020(n=77)(p<0.001)$ (Table 3$)$.

\section{Emergency operations}

Emergency operations decreased by $22 \%$ from $2019(n=1868)$ to $2020 \quad(n=1448)$ $(p<0.001)$ (Table 2). Trauma-related operations decreased by $42 \%$ ( $n=325$ v. $n=190 ; p<0.001)$. Appendicectomies and breast and colorectal cancer operation volume did not change significantly between 2019 and 2020 (Table 3).

\section{Surgical backlog}

The surgical backlog for elective operations from these six government hospitals for the 4-month study period was 1017 operations. The surgical backlog from each hospital ranged from 101 to 294 operations. This will take an estimated 4.6 - 13.4 months to address if each hospital can do an additional daily procedure during weekdays (Table 4).

\section{Discussion}

The study demonstrated that elective operations decreased by $\sim 75 \%$ and total general surgery operations by $>40 \%$ in the first 4 months of the COVID-19 pandemic in the Western Cape. The operative volume of laparoscopic cholecystectomy, a common general surgery operation, decreased by more than half. In just six hospitals, this represents a surgical backlog of $>1000$ elective operations over the first 4 months of COVID-19 lockdown. If our results are extrapolated nationwide, the effect of the surgical backlog is devastating.

The effect of COVID-19 on emergency procedures was most striking in traumarelated operation volumes. Lockdown significantly restricted non-essential movement, which was probably a major factor. In addition, for at least half of the 2020 study period there was a prohibition on alcohol, which is likely to have contributed to the decrease in the national trauma burden. Other studies from SA hospitals have also reported decreases in trauma cases. ${ }^{[7-9]}$

\begin{tabular}{lll}
\multicolumn{2}{l}{ Table 1. Study hospital characteristics } & \\
\hline Hospital & Hospital level & Beds, $\boldsymbol{n}$ \\
\hline New Somerset & Regional & 330 \\
Worcester & Regional & 227 \\
Karl Bremer & District & 273 \\
Mitchell's Plain & District & 230 \\
Khayelitsha & District & 230 \\
Eerste Rivier & District & 124
\end{tabular}

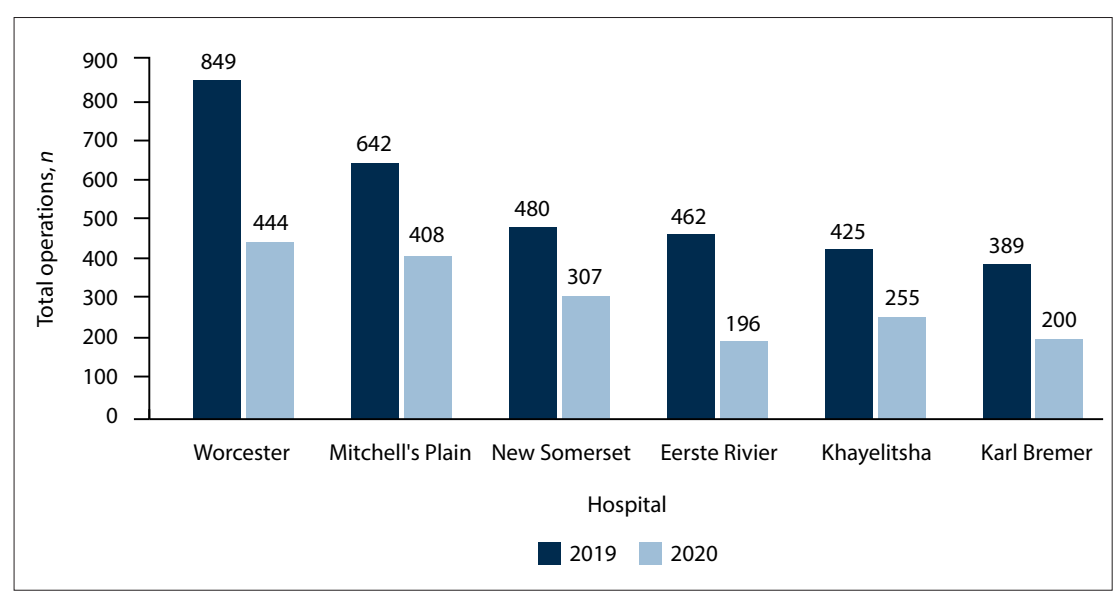

Fig. 1. General surgery operative volume from six South African hospitals, 2019 and 2020. 
Table 2. Comparison of operation volume for the six study hospitals, 2019 and 2020

\begin{tabular}{|c|c|c|c|c|c|}
\hline & \multicolumn{2}{|c|}{ Year, $n$} & \multirow[b]{2}{*}{ IRR } & \multirow[b]{2}{*}{ 95\% CI } & \multirow[b]{2}{*}{$p$-value } \\
\hline & $2019^{\dagger}$ & 2020 & & & \\
\hline Total operations & 3247 & 1810 & 0.56 & $0.53-0.59$ & $<0.001^{*}$ \\
\hline Total elective operations & 1379 & 362 & 0.27 & $0.24-0.30$ & $<0.001^{*}$ \\
\hline Worcester & 418 & 124 & 0.30 & $0.25-0.36$ & $<0.001^{*}$ \\
\hline Mitchell's Plain & 150 & 46 & 0.31 & $0.22-0.43$ & $<0.001^{*}$ \\
\hline New Somerset & 184 & 69 & 0.37 & $0.28-0.49$ & $<0.001^{*}$ \\
\hline Eerste Rivier & 311 & 73 & 0.23 & $0.18-0.30$ & $<0.001^{*}$ \\
\hline Khayelitsha & 134 & 33 & 0.24 & $0.17-0.36$ & $<0.001^{*}$ \\
\hline Karl Bremer & 182 & 17 & 0.09 & $0.06-0.15$ & $<0.001^{*}$ \\
\hline Total emergency operations & 1868 & 1448 & 0.78 & $0.72-0.83$ & $<0.001^{*}$ \\
\hline Worcester & 444 & 320 & 0.74 & $0.64-0.86$ & $<0.001^{*}$ \\
\hline Mitchell's Plain & 492 & 362 & 0.73 & $0.64-0.84$ & $<0.001^{*}$ \\
\hline New Somerset & 296 & 238 & 0.80 & $0.68-0.95$ & $0.012^{*}$ \\
\hline Eerste Rivier & 151 & 123 & 0.81 & $0.64-1.03$ & 0.091 \\
\hline Khayelitsha & 291 & 222 & 0.76 & $0.64-0.91$ & $0.002^{\star}$ \\
\hline Karl Bremer & 207 & 183 & 0.88 & $0.72-1.08$ & 0.225 \\
\hline 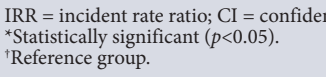 & & & & & \\
\hline
\end{tabular}

Table 3. Comparison of select elective and emergency operation volumes for the six study hospitals, 2019 and 2020

\begin{tabular}{|c|c|c|c|c|c|}
\hline & \multicolumn{2}{|c|}{ Year, $n$} & \multirow[b]{2}{*}{ IRR } & \multirow[b]{2}{*}{ 95\% CI } & \multirow[b]{2}{*}{$p$-value } \\
\hline & $2019^{\dagger}$ & 2020 & & & \\
\hline \multicolumn{6}{|l|}{ Select elective operation } \\
\hline Laparoscopic cholecystectomy & 242 & 77 & 0.32 & $0.24-0.41$ & $<0.001^{*}$ \\
\hline \multicolumn{6}{|l|}{ Select emergency operations } \\
\hline Traumatic injury & 325 & 190 & 0.58 & $0.49-0.70$ & $<0.001^{*}$ \\
\hline Appendicectomy & 276 & 255 & 0.92 & $0.78-1.10$ & 0.362 \\
\hline Breast cancer & 112 & 91 & 1.21 & $0.93-1.62$ & 0.141 \\
\hline Colorectal cancer & 65 & 71 & 0.78 & $0.78-1.53$ & 0.607 \\
\hline
\end{tabular}

Table 4. Estimated surgical backlog for elective operations from the six study hospitals during the first 4 months of COVID-19 in 2020

\begin{tabular}{lll}
\hline Hospital & Case backlog, $\boldsymbol{n}$ & $\begin{array}{l}\text { Months to } \\
\text { address backlog }\end{array}$ \\
\hline Worcester & 294 & 13.4 \\
Eerste Rivier & 238 & 10.8 \\
Karl Bremer & 165 & 7.5 \\
New Somerset & 115 & 5.2 \\
Mitchell's Plain & 104 & 4.7 \\
Khayelitsha & 101 & 4.6
\end{tabular}

The study showed that the volume and proportion of non-trauma emergency surgical conditions were less affected by COVID-19 policies than elective and trauma conditions. Appendicectomy, the most common non-trauma general surgery emergency procedure, demonstrated only a marginal decrease between 2019 and 2020. Studies from other countries have reported up to $40 \%$ decreases in appendicectomy volume, ${ }^{[10,11]}$ as well as an increase in the severity of acute appendicitis presentation. ${ }^{[12]}$ While we did not measure severity of disease, our results suggest that care for non-trauma emergency surgical conditions remained accessible in SA despite a stricter lockdown than in other countries.

Treatment for breast and colorectal cancer is primarily surgical, and delays can result in increased mortality. This study did not demonstrate a decrease in surgical access for these conditions. Even though access to outpatient elective surgery clinics and screening was dramatically decreased, the proportion of obstructed and perforated colorectal cancer cases did not increase. Other studies have reported the need to balance the risk of contracting COVID-19 for cancer patients while hospitalised with the need to push for continued oncological surgical services, given the risk of worsening outcomes due to treatment delays. ${ }^{[13,14]}$ Further studies are needed to measure the longer-term effects of the COVID-19 pandemic on stage of cancer presentations.

This study is unique because it confirms, with primary data, previous modelling studies, surveys and anecdotal reports, that access to elective surgical care during the COVID-19 lockdown in SA was reduced. ${ }^{[2-4]}$ These findings can be used to estimate the surgical backlogs of different operation types and help hospital managers and policymakers plan ways to increase post-pandemic access. Our 
study shows that if hospitals can do an additional general surgery operation each day, it will take between 4 and 14 months to clear the backlog. This estimate is optimistic because with vaccines not yet widely available, the pandemic is unlikely to subside for months or even years in SA, and operative services will be functioning below capacity well into 2021 . With additional backlogs therefore likely, new tools are needed to triage elective surgical patients. The SA National Department of Health has endorsed a COVID-19 risk calculator adopted for the local context from the MENTS scoring system for elective surgery triage. ${ }^{[15,16]}$ However, this does not address the surgical backlog. Novel strategies to increase surgical access above pre-COVID-19 times once the pandemic has slowed are needed. Shared backlog lists between hospitals, both public and private, could be a co-ordinated and possibly more equitable way to address the unmet burden of disease. ${ }^{[2]}$ Specifically, it would promote interfacility engagement and boost network referrals between hospitals, for sharing lessons learned during this pandemic. In addition, a platform for virtual consultation between patients and healthcare workers can be advocated for. ${ }^{[2]}$ Additional operation lists could be one potential solution. Both health sectors working together to improve surgical access will also be the goal of the upcoming National Health Insurance plan.

\section{Study limitations}

This study had several limitations. We measured changes in surgical volume but no other components of surgical care such as inpatient admissions and outpatient clinics, which could also have been affected by the COVID-19 pandemic. The classification of operation types (such as emergency and elective) was done by each hospital team and may have been inconsistent across study sites. However, intrahospital classification by year was probably consistent, thereby having a minimal effect on the change in operative volume. In addition, the surgical backlog is probably an underestimation because some emergency surgical conditions (such as cancer) may still be awaiting definitive surgical treatment but were not included in our calculation. However, given that the three non-trauma emergency conditions we evaluated were not significantly different between 2019 and 2020, it is hoped that this underestimation is minimal. Finally, this study only considered general surgery operations, and the results may not be translatable to other surgical subspecialties. However, the strength of this study is that we examined primary data from six government hospitals in one SA province, which consistently demonstrated an overall decrease in elective operative volume.

\section{Conclusions}

Access to surgical health services in SA has become collateral damage to the pandemic. This study has shown that COVID-19 has created large backlogs of elective operations that will need to be addressed urgently. Clear and structured guidelines need to be developed in order to streamline the re-introduction of full surgical healthcare services.

\section{Declaration. None.}

Acknowledgements. The authors would like to acknowledge all the frontline workers in the COVID-19 pandemic in South Africa.

Author contributions. KMC and RD conceived the study design. CPS, LB, $\mathrm{HB}, \mathrm{RD}, \mathrm{AG}, \mathrm{LvdW}$ and AEV collected the data. KMC and JM conducted the data analysis. All authors approved the final version.

Funding. None.

Conflicts of interest. None.

1. World Health Organization. WHO Coronavirus (COVID-19) Dashboard. https://covid19.who.int/ region/afro/country/za (accessed 1 February 2021).

2. Biccard B, Cairncross L. Over 70\% of surgeries in SA will be cancelled or postponed due to Covid-19how will we catch up? Daily Maverick, 31 May 2020. https://www.dailymaverick.co.za/article/2020-0531-over-70-of-surgeries-in-sa-will-be-cancelled-or-postponed-due-to-covid-19-how-will-we-catchup/ (accessed 5 December 2020).

3. Chu KM, Smith M, Steyn E, Goldberg P, Bougard H, Buccimazza I. Changes in surgical practice in 85 South African hospitals during COVID-19 hard lockdown. S Afr Med J 2020;110(9):916-919. https:// doi.org/10.7196/SAMJ.2020.v110i9.15014

4. COVIDSurge Collaborative. Elective surgery cancellations due to the COVID-19 pandemic: Global predictive modelling to inform surgical recovery plans. Br J Surg 2020;107(11):1440-1449. https://doi. org/10.1002/bjs.11746

5. Makatini GM, Mewa Kinoo S, Singh B. An audit of interval cholecystectomy for acute cholecystitis in Makatini GM, Mewa Kinoo S, Singh B. An audit of interval cho
a low resource healthcare system. S Afr J Surg 2020;58(1):10-13

6. Mbatha SZ, Anderson F. Outcomes in laparoscopic cholecystectomy in a resource constrained environment. S Afr J Surg 2016;54(3):8-12.

7. Moustakis J, Piperidis AA, Ogunrombi AB. The effect of COVID-19 on essential surgical admissions in South Africa: A retrospective observational analysis of admissions before and during lockdown at a tertiary healthcare complex. S Afr Med J 2020;110(9):910-915. https://doi.org/10.7196/SAMJ.2020. v110i9.15025

8. Reuter H, Jenkins LS, de Jong M, Reid S, Vonk M. Prohibiting alcohol sales during the coronavirus disease 2019 pandemic has positive effects on health services in South Africa. Afr J Prim Health Care disease 2019 pandemic has positive effects on health services in South
Fam Med 2020;12(1):e1-e4. https://doi.org/10.4102/phcfm.v12i1.2528

9. Venter A, Lewis CM, Saffy P, Chadinha LP. Locked down: Impact of COVID-19 restrictions on trauma presentations to the emergency department. S Afr Med J 2020;111(1):52-56. https://doi.org/10.7196/ SAMJ.2021.v111i1.15289

10. Neufeld MY, Bauerle W, Eriksson E, et al. Where did the patients go? Changes in acute appendicitis presentation and severity of illness during the coronavirus disease 2019 pandemic: A retrospective cohort study. Surgery 2021;169(4):808-815. https://doi.org/10.1016/j.surg.2020.10.035

11. Tankel J, Keinan A, Blich O, et al. The decreasing incidence of acute appendicitis during COVID-19: A retrospective multi-centre study. World J Surg 2020;44(8):2458-2463. https://doi.org/10.1007/s00268020-05599-8

12. Snapiri O, Rosenberg Danziger C, Krause I, et al. Delayed diagnosis of paediatric appendicitis during the COVID-19 pandemic. Acta Paediatr 2020;109(8):1672-1676. https://doi.org/10.1111/apa.15376
the

13. Elanko A, Khan J, Hamady ZZ, Malik H. Cancer surgery sustainability in the light of COVID-19 pandemic. Eur J Surg Oncol 2020;46(6):1174-1175. https://doi.org/10.1016/j.ejso.2020.05.018

14. Finley C, Prashad A, Camuso N, et al. Guidance for management of cancer surgery during the COVID-19 pandemic. Can J Surg 2020;63(22):S2-S4. https://doi.org/10.1503/cjs.005620

15. Department of Health, Republic of South Africa. Internal memo: Guide for non-emergency surgery during the Covid-19 pandemic. 28 August 2020. https://sacoronavirus.co.za/wp-content/ uploads/2020/10/MAC-Advisory-Memo_Non-Emergency-Surgery_28-August-2020.pdf (accessed 30 October 2020).

16. Chu K, Owolabi E, Smith M, et al. Establishing a South African national framework for COVID-19 surgical prioritisation. S Afr Med J 2021;111(5):426-431. https://doi.org/10.7196/SAMJ.2021. v111i5.15603

Accepted 28 April 2021. 